\title{
Nonlinear effects in gases due to strong gradients ${ }^{1}$
}

\author{
Patricio Cordero* and Dino Risso ${ }^{\dagger}$ \\ * Departamento de Física, Universidad de Chile, Santiago, Chile \\ ${ }^{\dagger}$ Departamento de Física, Universidad del Bí-Bío, Concepción, Chile
}

\begin{abstract}
The behavior of a gas may be quite atypical if the velocity distribution function is significantly distorted by the presence of large gradients. Under such circumstances phenomena like shear thinning or negative effective thermal conductivity may be present. Hydrodynamic equations derived directly from Boltzmann's equation using a moment expansion method yield predictions which are sensitive to the value of Knudsen numbers associated to the macroscopic fields themselves and provide a rational for the peculiar behavior. In the case of sheared laminar flows the law of viscous flow predicts non-Newtonian effects including shear thinning and the law of energy transport (associated to what is usually called heat flux) is more general than Fourier's law: it is not linear and it implies a flux with a nontrivial component parallel to the isotherms. These nonlinear transport laws are well corroborated by molecular dynamic simulations based on straightforward Newtonian dynamics. More in general it is shown that the energy flux is naturally split in a component that removes heat from the system and a divergenceless component which represents a flux of energy which does not leave the system.
\end{abstract}

\section{INTRODUCTION}

Extracting information from Boltzmann's equation can be a subtle and difficult endeavor [1,2]. The integrodifferential nature of Boltzmann's equation has a free convective part on the left hand side and a collisional term on the right hand side. Rarefied gases are sometimes identified with those for which the collisional term can be considered a perturbation of the free convective part, which is tantamount to saying that the Knudsen number - defined as the ratio between the mean free path and the typical distance $L$ between walls, Kn $\sim \ell / L-$ is comparable to unity or larger. Under such circumstances no standard hydrodynamics can be valid. There are other physical situations under which standard hydrodynamics cannot be valid. For example, associating a distance $L_{X}=X_{0} /|\nabla X|$, to a inhomogeneous hydrodynamic field $X\left(X_{0}\right.$ being a typical value for $\left.X\right)$, if the Knudsen number

$$
K_{X}=\ell / L_{X}
$$

is not negligible, standard hydrodynamics cannot hold either.

Standard hydrodynamics is built with three types of equations. For example it takes the form of: (i) dynamic equations for the number density $n$, the velocity field $\mathbf{v}$ and temperature field $T$; (ii) equations of state for the energy density $u=u(n, T)$ and the hydrostatic pressure $p=p(n, T)$ and (iii) constitutive equation that take the form of two linear transport laws: Newton's law of viscous flow and Fourier's law of heat conduction [3]. When Chapman-Enskog method is used to solve Boltzmann's equation the previous picture is recovered in the so called Navier-Stokes level [2]. Beyond that the complex Burnett scheme is obtained. None of these schemes are enough to describe the regimes we have been studying.

All this picture gets invalidated if at least one field $X$ has a gradient large enough that the effects stemming from the associated Knudsen number $K_{X}$ cannot be neglected. This is true even though the standard Knudsen number Kn continues being small.

1) URL: http://www.cec.uchile.cl/cinetica/ 
In the following sections we describe the way to get a gas dynamics from a moment expansion. The present article then analyzes the case of sheared gases, describe a theoretical framework specialized for them, compare some of its implications with our own Newtonian molecular dynamic results and give a viewpoint from which the energy flux (sually called heat flux) can be split in two physically different vector fields: $\mathbf{Q}_{\|}$is a flux carrying energy from hot to cold parts and $\mathbf{Q}_{\perp}$ is a flux of energy moving in closed circuits inside the system, never leaving it. Usual hydrodynamics assumes that only $\mathbf{Q}_{\|}$exists but rarefied gas physics has known for quite some time now that there is something else. For example, see the remark after (8.20) in [4] and the top right paragraph in page 892 in [5]. The words hot and cold are written in a slanted form, because for gas systems far from equilibrium it is not universal what one should understand by temperature. In the present paper temperature $T$ is used to mean the average kinetic energy per particle, but we will see that $\mathbf{Q}_{\|}$does not obey a law directly related to the gradient of $T$, but rather to $-\nabla \Theta$ and $\Theta$ cannot be expressed in any simple way in terms of the hydrodynamic fields.

\section{GAS DYNAMICS}

The theory described below comes from the picture given originally by Grad [6]. It is a moment expansion which is known from plain statistics to be a powerful method. Moment expansion methods can summarily be described as follows. The local Maxwellian distribution function $f_{M}(\mathbf{r}, \mathbf{c}, t)$ is considered to be the reference function about which an expansion is made. It is written in terms of the peculiar velocity $\mathbf{C}=\mathbf{c}-\mathbf{v}(\mathbf{r}, t)$, where $\mathbf{v}(\mathbf{r}, t)$ is the hydrodynamic velocity. A set of orthonormal polynomials on $\mathbf{C}, H_{a}(\mathbf{C})$, are built in the sense that $H_{0}=1$ and

$$
\int H_{a}(\mathbf{C}) H_{b}(\mathbf{C}) f_{M}(\mathbf{r}, \mathbf{C}, t) d \mathbf{C}=\delta_{a b} .
$$

The polynomials $H_{a}$ are obtained simply by building a base of orthonormal polynomials starting from $H_{0}=1$ and from first degree upwards using standard methods. We have built polynomials only on $C_{i}, C_{i} C_{j}$ and $C^{2} C_{i}$ as Grad did, namely, up to third order and not all of them. One could go on but so far this seems to be enough. The resulting polynomials $H_{a}$ are Hermite polynomials. Next, the distribution function is defined having the form

$$
f(\mathbf{r}, \mathbf{C}, t)=\sum_{a} H_{a}(\mathbf{C}) R_{a}(\mathbf{r}, t) f_{M}(\mathbf{r}, \mathbf{C}, t)
$$

The coefficients $R_{a}$ (moments of the distribution) are formally obtained requiring first that $f$ is normalized to the number density: $\int f d \mathbf{c}=n$ and then that the averages $\langle A\rangle \equiv \frac{1}{n} \int A d \mathbf{c}$ give the formally correct results, namely, it is required that the hydrodynamic velocity is $\mathbf{v}=\langle\mathbf{c}\rangle$, the temperature is $T=\frac{m}{2}\left\langle C^{2}\right\rangle$ (the temperature is measured in energy units so that Boltzmann's constant is $k_{B}=1$.), the pressure tensor is $P_{i j}=\left\langle m n C_{i} C_{j}\right\rangle$ and the heat flux vector is $\mathbf{Q}=\frac{m n}{2}\left\langle C^{2} \mathbf{C}\right\rangle$. Such requirements totally define the $R_{a}$. In such formalism the collisional contribution to transport is neglected altogether.

The following step is to replace the above distribution in Boltzmann's equation to derive integrability conditions multiplying the kinetic equation consecutively by the $H_{a}$ and integrating the equation over $\mathbf{C}$. The idea is to do this up to a given order and drop all contributions coming from polynomials of degree higher than a chosen value (up to order 3 in our case). These integrability conditions turn out to be a set of hydrodynamic equations for the different moments.

The resulting gas-dynamic equations were found by Grad [6] and they, when specialized to the $d$-dimensional fluid of hard spheres $(d=2$ or 3$)$, can be found in [7,8]. This gas-dynamics consists not only of equations for the density, the velocity field and the temperature, but it also consists of equations for the symmetric traceless part $p_{i j}$ of the pressure tensor $\left(P_{i j}=p \delta_{i j}+p_{i j}\right)$, and for the heat flux vector $\mathbf{Q}$. The extra equations are, we reiterate, dynamic equations and they take the place of the constitutive equations in standard hydrodynamics. Namely, there is no need to introduce constitutive equations and their role is taken by dynamic equations which enter the formalism in the same footing as the traditional hydrodynamic equations.

The moment expansion method as used in the present work assumes analyticity of the distribution function. It is known that this is not true near hard walls. Hence the present formalism cannot be expected to be true near walls. 


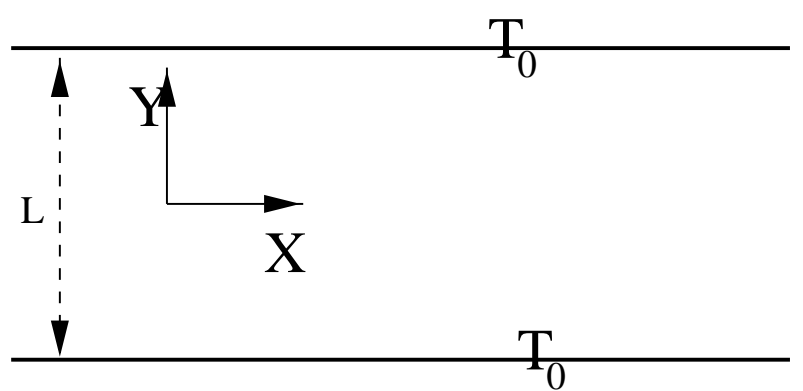

FIGURE 1. A flat channel of width $L$ and walls parallel to the $X$ axis kept at temperature $T_{0}$. The laminar flow is in the $X$ direction $\left(v_{y}=0\right)$ and all hydrodynamic fields depend only on the transversal coordinate $y$.

\section{LAMINAR FLOW}

We have been studying this dynamics in the case of laminar planar flows with the velocity field having a gradient large enough that its effect cannot be described using standard hydrodynamics. Below we give the generic equations for a laminar, planar and stationary bidimensional flow in the $X$ direction in the presence of an external force ( $X$ direction as well) which, on each particle of the fluid, is $m g$. Only the component $v_{x}(y)$ is nontrivial (see Fig. 1) and with it we define the dimensionless shear rate $\gamma$

$$
\gamma=\tau \frac{d v_{x}}{d y}
$$

where $\tau \equiv \frac{1}{2 \sigma p} \sqrt{\frac{m T}{\pi}}$ is the local relaxation time, $\sigma$ is the diameter of the hard particles and the only relevant coordinate is the transversal coordinate $y$. It turns out that the Knudsen number $K_{v},\left(K_{X}\right.$ in the introduction) associated to the velocity $v_{x}$ is $\gamma$ except for $\mathcal{O}(1)$ factors, namely, the dimensionless shear rate can be considered the Knudsen number associated to the velocity field and its gradient.

From now on, instead of using the traceless and symmetric part of the pressure tensor, $p_{i j}$ we use some of the components of $P_{i j}=p \delta_{i j}+p_{i j}$ as the independent hydrodynamic fields. The formalism yields, in this laminar stationary case, hydrodynamic equations for the following seven fields: $n, \gamma, T, P_{y y}, P_{x y}, Q_{x}$ and $Q_{y}$

$$
\begin{aligned}
P_{y y} & =\text { uniform } \\
P_{x y}^{\prime} & =\frac{m g}{T} P_{y y}-\frac{3 m g \gamma}{T} P_{x y} \\
\tau Q_{y}^{\prime} & =-\gamma P_{x y} \\
-\frac{\tau}{2} Q_{x}^{\prime} & =\gamma P_{y y}-\gamma p+(p \tau) v_{x}^{\prime}+P_{x y} \\
p & =P_{y y}-\frac{3 \gamma}{2} P_{x y}, \\
Q_{y} & =-\frac{4 \tau P_{y y}}{m} T^{\prime}+g \tau\left(2 P_{x y}-3 \gamma p\right)-\gamma Q_{x}-\frac{3 \tau P_{x y}}{m}(\gamma T)^{\prime} \\
Q_{x} & =3 \gamma Q_{y}+2 g \tau P_{y y}+\frac{6 \tau P_{x y}}{m} T^{\prime}
\end{aligned}
$$

where the prime denotes derivative with respect to $y$. The mass continuity equation is an identity. The first two equations

are the momentum balance equations and in Eq. (2) the term proportional to $P_{x y}$ is characteristic of the present approach. If there is no external force $\mathbf{g}$ this equation tells us that $P_{x y}$ is uniform too; this is the case of 

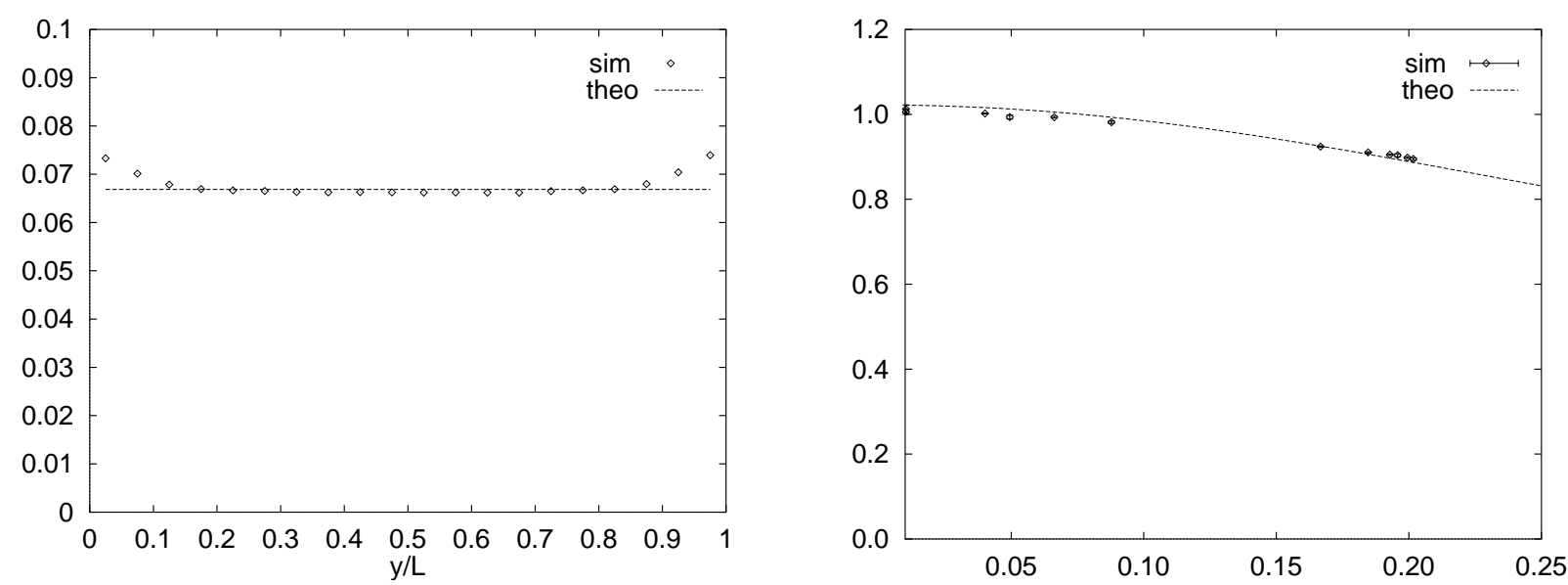

FIGURE 2. At left the profile of the dimensionless shear rate $\gamma$ observed in a Newtonian molecular dynamic simulation for a system with $N=7680$ particles, fraction of occupied area $\rho_{\mathrm{A}}=0.01$ and $v_{0}=1.4 \sqrt{T_{0} / m}$. The horizontal line represents the theoretical value $\gamma=0.066869 \pm 0.000062$. Away from the walls the discrepancy is about of $0.9 \%$. At right the viscosity ratio $\eta / \eta_{0}$ given in (8) versus $\gamma$ showing shear thinning, is compared with the viscosity ratio derived directly from the observations of a Couette flow of a system of $N=7680$ particles.

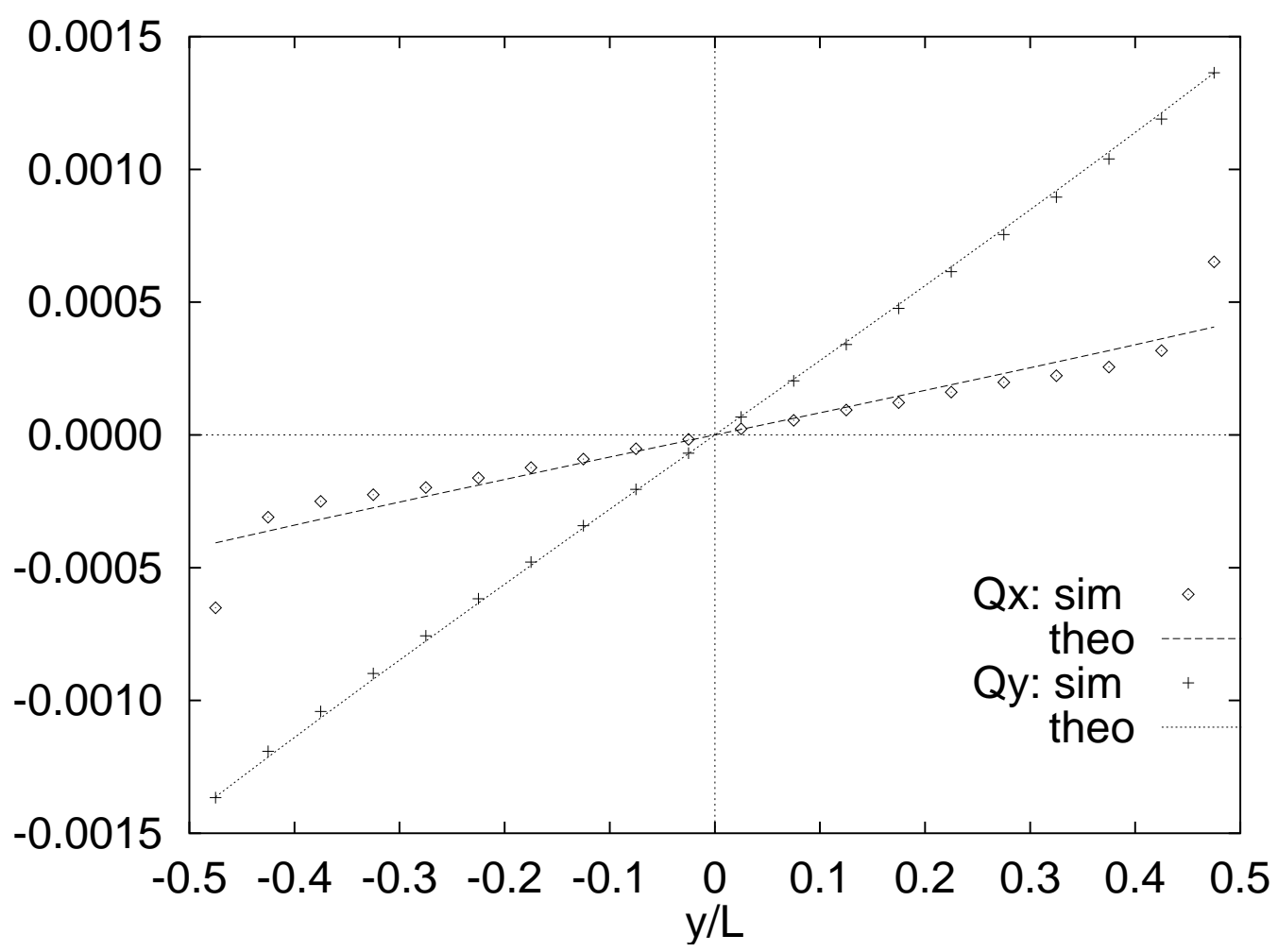

FIGURE 3. The theoretical and observed profiles of the heat currents $\mathbf{Q}$ for the system with $N=7680$ particles and $\gamma=-0.066869 \pm 0.000062$. The transversal heat current $Q_{y}$ (crosses) fits quite well the theory, while the agreement between the theoretical and observed profiles of the longitudinal heat current $Q_{x}$ (rhombus) is fair. Both currents are scaled with the factor $T_{0} \sqrt{T_{0} / m}$. 


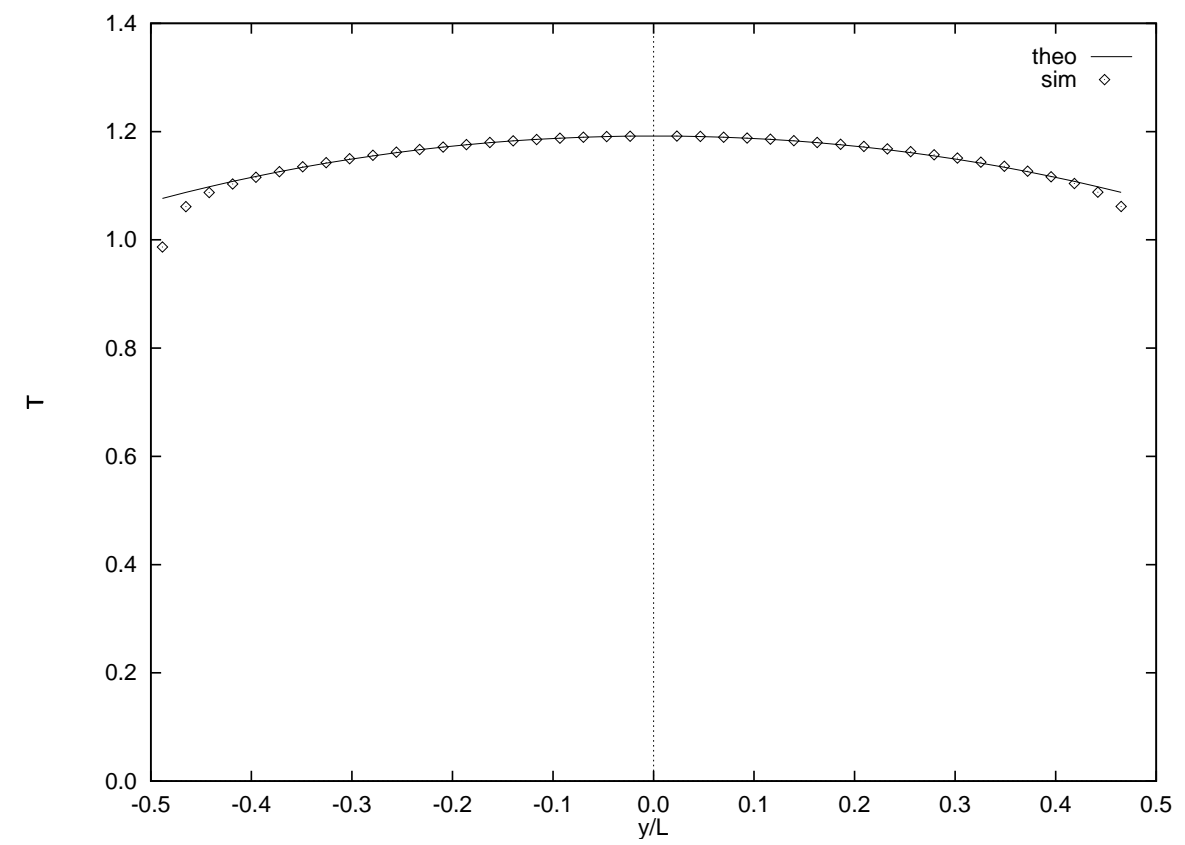

FIGURE 4. Temperature profile for a Couette system with $T_{0}=1, \gamma=0.06$ and $N=29538$ particles. The rhombus give the simulational results and the solid line corresponds to the theoretical prediction. Notice that the temperature near the walls is distinctly not equal to $T_{0}=1$.

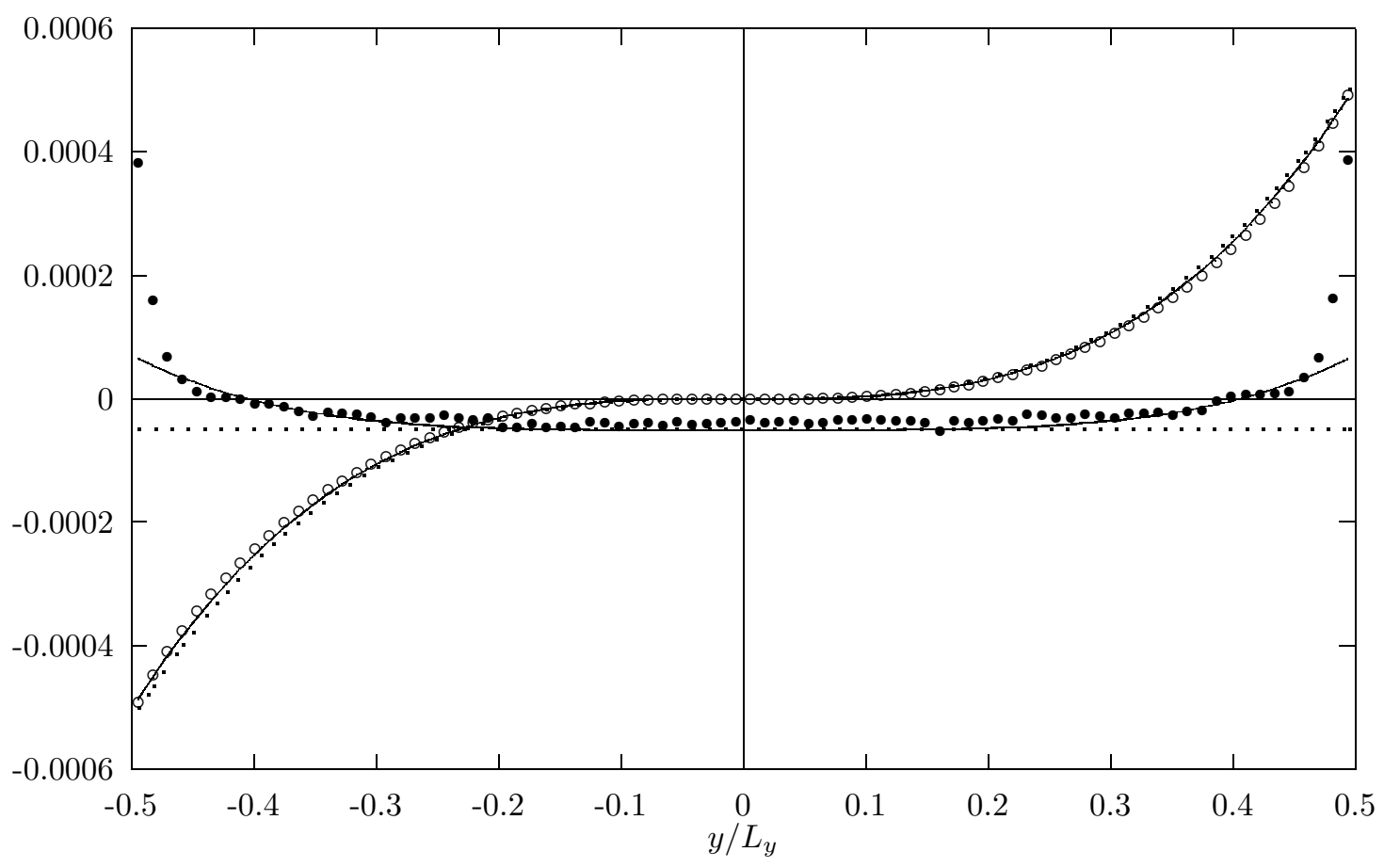

FIGURE 5. The theoretical and observed profiles of the heat currents $\mathbf{Q}$ of a Poiseuille system of $N=7056$ particles, fraction of occupied area $\rho_{A}=0.01$ 
a Couette flow, for example. The third equation is the standard energy balance equation. If in Eq. (4) all terms are erased except for the last two, Newton's usual viscous flow law is recovered, but Eq. (4) in full is a quite nontrivial transport law which involves the heat flux component $Q_{x}$ parallel to the isotherms. Equations (5) come from the balance associated to the $p_{i j}$ and they provide algebraic relations for the hydrostatic pressure and for $P_{x x}$ in terms of $P_{x y}$ and $P_{y y}$. The fact that $P_{x x}$ differs from $P_{y y}$ is a characteristic feature of the local anisotropy of the present gas regime. The first two terms of Eq. (6) alone would give Fourier's law but again, the present law is quite nonlinear and it involves other fields [9]. The last equation is an equation for $Q_{x}$ and it is absent from standard hydrodynamics. This flux coincides with remarkable precision with the one observed in our molecular dynamic simulations.

In [8] we were able to find a closed solution to this hydrodynamics in the case of a laminar planar Couette flow $(g=0)$ characterized by externally imposed velocities $\pm v_{0}$ at the two opposite walls in Fig. 1 . In this case the formalism predicts that $\gamma$ is uniform which can be compared with the simulational results shown in Fig. 2: only near the walls, where the present theory is known to be unreliable, the uniformity of $\gamma$ clearly fails. In [8] it is shown, for example, a closed and nonlinear law of viscous flow that can be cast in the form of Newton's law but with a shear dependent viscosity coefficient

$$
\frac{\eta}{\eta_{0}}=\frac{8}{15 \gamma^{2}+4+\sqrt{16+120 \gamma^{2}-63 \gamma^{4}}}
$$

where $\eta_{0}$ is the well known ideal gas shear viscosity, $\eta_{0}=\sqrt{m T / \pi} /(2 \sigma)$. It is seen that $\eta$ depends on the shear rate $\gamma$ and presents shear thinning as seen in Fig. 2 both the predicted and observed values. The observed and predicted values for the two components of the heat flux are seen in Fig. 3. Those results lead us to get analytic closed forms for the thermal conductivities associated to $Q_{x}$ and to $Q_{y}$ [8]. They depend not only on temperature and density but also, like the effective viscosity, they depend on the shear rate. The temperature seems to be sensitive to the size of the system up to relatively large systems and we had to go to a system of about 30000 particles to obtain a good fit, shown in Fig. 4.

Similar results have also been obtained for a laminar stationary Poiseuille flow [10] except for one interesting feature predicted by our formalism and observed in our simulations: even though generally speaking the system is hotter in the middle of the channel and the heat flows towards the walls, the temperature profile $T$ has a shallow minimum at the center as shown in Fig. 6 in spite that the heat flux vector takes the energy towards the walls. This minimum has been observed in simulations and an explanation was originally given using the BGK approximation to Boltzmann equation [11], see also [10]. Namely, near the center of the channel the system behaves as if the thermal conductivity was negative as in [5]. The dimensionless quantity which serves to measure the intensity of the external force in this case is seen to be $\mathcal{F}=m g L /\left(T_{0} \mathrm{Kn}\right)$, where $L$ is the width of the Poiseuille channel. Our formalism says that this local minimum exists for all values of $\mathcal{F}$. The solution found in [10] is perturbative using $\mathcal{F}$ as small parameter, hence Kn cannot be too small. In the next section we comment on this and other peculiarities of the energy (heat?) flux.

\section{PECULIARITIES OF THE ENERGY FLUX}

Our aim is to analyze the meaning and physical reason for the existence of an energy flux $\mathbf{Q}$ which is not totally pointing in the direction of $-\nabla T$. It is quite general that a vector field can be split in the form

$$
\mathbf{Q}=\mathbf{Q}_{\|}+\mathbf{Q}_{\perp}
$$

in such a way that

$$
\begin{array}{lll}
\nabla \times \mathbf{Q}_{\|}=0 & \Longleftrightarrow & \mathbf{Q}_{\|}=-\nabla \Theta \\
\nabla \cdot \mathbf{Q}_{\perp}=0 & \Longleftrightarrow \quad \mathbf{Q}_{\perp}=\nabla \times \mathbf{A}
\end{array}
$$

This split is not unique but it always exists. For example, finding a vector field $\mathbf{R}$ by solving $\nabla^{2} \mathbf{R}=-\mathbf{Q}$ leads to define

$$
\mathbf{A}=\nabla \times \mathbf{R} \quad \text { and } \quad \Theta=\nabla \cdot \mathbf{R}
$$



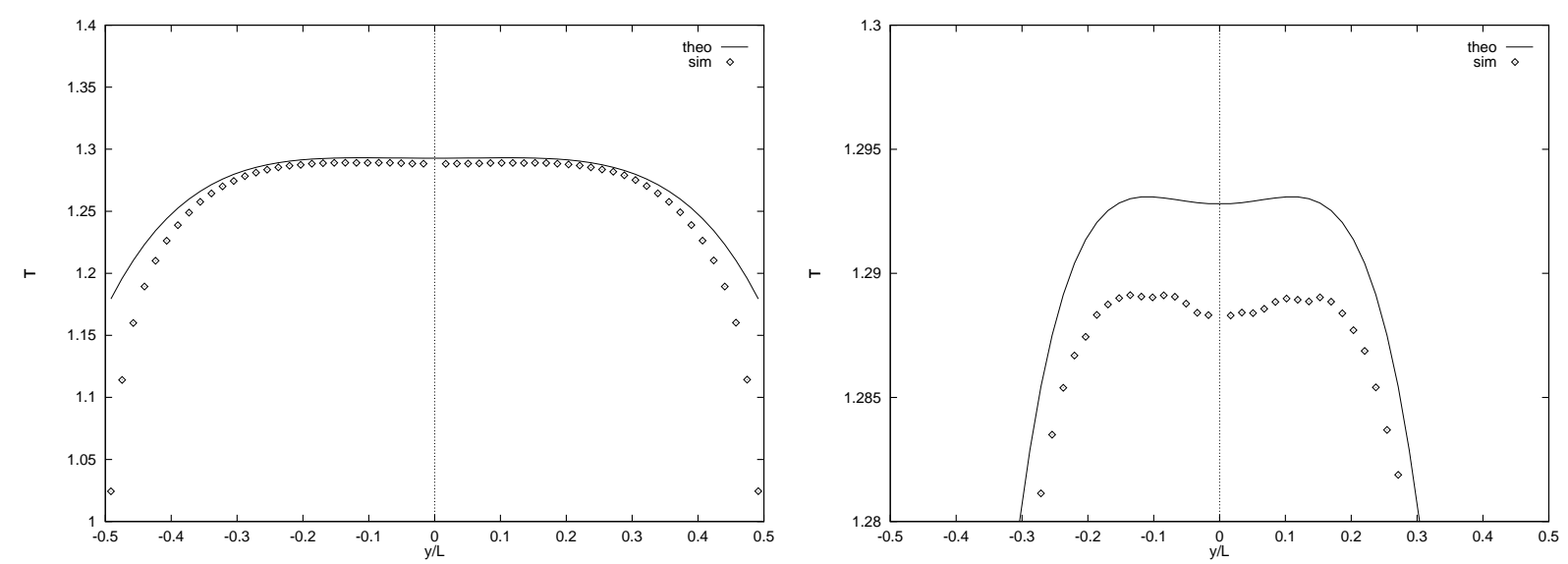

FIGURE 6. At left the temperature profile in the case of a system Poiseuille flow obtained with a system with $N=9581$ particles, $\mathcal{F}=2.5195$ and fraction of occupied area, $\rho_{A}=0.005$ and $\mathrm{Kn}=2 /(\sqrt{\pi} \sigma n L)=0.101$. The figure at right amplifies the central part of the profile.

In fact, it can easily be seen that there is consistency using the following check:

$$
\mathbf{Q}_{\perp}=\nabla \times(\nabla \times \mathbf{R}) \equiv \nabla(\nabla \cdot \mathbf{R})-\nabla^{2} \mathbf{R}=-\mathbf{Q}_{\|}+\mathbf{Q}
$$

From the physical point of view the divergenceless nature of $\mathbf{Q}_{\perp}$ means that it represents a flux along closed lines (no sources) showing that $\mathbf{Q}_{\perp}$ represents an energy flux in the system without ever leaving it. With $Q_{\perp}$ one could define lines of flux resembling a textbook description of a magnetic field. In contrast $\mathbf{Q}_{\|}$, like a conservative force, or like in Fourier's law, can be expressed as the gradient of a scalar field and an integration $\int_{a}^{b} \mathbf{Q}_{\|} \cdot d \mathbf{r}$ gives a unique difference $\Theta_{a}-\Theta_{b}$ regardless of the integration path. The field $\Theta$ resembles a temperature, but dimensionally it is like $T^{3 / 2}$.

In the case of the planar Couette flow taking

$$
\mathbf{A}=\left(\begin{array}{c}
0 \\
0 \\
\alpha_{A} T^{3 / 2}(y)
\end{array}\right), \quad \quad \Theta=\alpha_{\theta} T^{3 / 2}(y)
$$

makes us recover the expressions for the heat flux vector found in [8] provided that the constants $\alpha_{k}$ are given by,

$$
\begin{aligned}
& \alpha_{\theta}=-\frac{16}{\sigma \sqrt{\pi}} \frac{1}{33 \gamma^{2}-4-\delta} \\
& \alpha_{A}=\frac{1}{24 \sigma \sqrt{\pi} \gamma^{3}} \frac{8-6 \gamma^{2}+9 \gamma^{4}-\left(2-9 \gamma^{2}\right) \delta}{1-3 \gamma^{2}}
\end{aligned}
$$

where $\delta \equiv \sqrt{16+120 \gamma^{2}-63 \gamma^{4}}$. As mentioned before, in this case in fact the dimensionless shear rate $\gamma$ is uniform and therefore the $\alpha_{k}$ 's are indeed constants.

At it was mentioned at the end of the last section, it has been observed that in the case of a planar laminar Poiseuille flow the temperature profile $T$ shows a shallow local minimum at the center of the channel and in fact the gas dynamics described by Eqs. (1)-(7) predicts that such local minimum exists. In Fig. 6 there is a comparison between theory and simulational results for a Poiseuille system with $\mathcal{F}=2.5195$. This peculiar behavior becomes more edible once we realize that the field $\Theta$ decreases monotonically from its maximum at the center. Namely, there is something like a Fourier law but it is $\Theta$ and not $T$ the field that drives the energy flux from $\Theta_{\text {hot }}$ to $\Theta_{\text {cold }}$ regions of the system. To be absolutely sure that $\Theta(y)$ has a maximum at the center of the channel and that it is a concave function all the way up to the walls we notice that $\Theta^{\prime \prime}=-Q_{y}^{\prime}<0$. In 
fact from Eq. (3) it is seen that $-Q_{y}^{\prime}=\gamma P_{x y} / \tau$ which is a negative quantity because $P_{x y}$ and $\gamma$ have opposite sign.

In summary, Boltzmann's equation plus moment expansion methods yield gas-dynamic equations beyond standard hydrodynamics. These are dynamic equations for: $n, \mathbf{v}, T, P_{i j}$ and $\mathbf{Q}$ and there is no need to assume constitutive equations whatsoever. Instead they imply nonlinear dynamic equations for $P_{i j}$ and $\mathbf{Q}$. Newtonian molecular dynamic simulations are excellently well described by such formalism (at least laminar flows). The non-Newtonian and non-Fourier behavior of gases (e.g., anomalous energy flux) should be measurable in actual experiments. The non-Fourier behavior has been analized showing that the flux $\mathbf{Q}$ naturally splits in components that extranct $\left(\mathbf{Q}_{\|}\right)$and do not extract heat from the system $\left(\mathbf{Q}_{\perp}\right)$. The first component satisfies a Fourier-like law. The other component is divergenceless and represents a flux of energy that circulates inside the system never abandoning it.

This work has been partially financed by Fondecyt grants 1000884 (P.C.) and 1990148 (D.R.).

\section{REFERENCES}

1. C. Cercignani, Mathematical Methods in Kinetic Theory (Plenum, New York/McMillan, London, 1969).

2. Ferziger J. H. and Kaper H.G. Mathematical Theory of Transport Processes in Gases, North-Holland 1972.

3. Chapman S. and Cowling T. G. The Mathematical Theory of Nonuniform Gases, (Cambridge University Press, third edition, 1970).

4. C. Cercignani, The Boltzmann Equation and Its Applications (Springer-Verlag, New York, 1988).

5. H.M. Mott-Smith, Phys. Rev. 82, 885 (1951).

6. H. Grad, Comm. Pure Appl. Math. 2, 331 (1949); H. Grad, Principle of the Kinetic Theory of Gases, in Handbuch der Physik, Vol. XII, edited by S. Flüge (Springer, Berlin, 1958).

7. P. Cordero and D. Risso, Microscopic Computer Simulation of Fluids in Fourth Granada Lectures on Computational Physics, P.L. Garrido and J. Marro, eds., (Springer-Verlag, 1996).

8. D. Risso and P. Cordero, Phys. Rev. E 56, 489 (1997); Erratum: Phys. Rev. E 577365 (1998).

9. P. Cordero and D. Risso, Physica A 25736 (1998).

10. D. Risso and P. Cordero, Phys. Rev. E 58, 546 (1998).

11. M. Malek-Mansour, F. Baras and A.L. García, Physcia A 240255 (1997); M. Tij, M. Sabbane and S. Santos, Phys. Fluids 10, 1021 (1998). 